\title{
Nano-Scale Gene Delivery Systems: Current Technology, Obstacles, and Future Directions
}

\author{
Antonio Garcia-Guerra ${ }^{1}$, Thomas L. Dunwell ${ }^{2}$ and Sonia Trigueros, ${ }^{1,2, *}$ \\ ${ }^{1}$ Department of Physics, University of Oxford, OX26GG Oxford, UK, ${ }^{2}$ Department of Zoology, University of Oxford, OX26GG \\ Oxford, UK
}

ART I CLE H IS T ORY

\begin{abstract}
Within the different applications of nanomedicine currently being developed, nanogene delivery is appearing as an exciting new technique with the possibility to overcome recognised hurdles and fulfill several biological and medical needs. The central component of all delivery systems is the requirement for the delivery of genetic material into cells, and for them to eventually reside in the nucleus where their desired function will be exposed. However, genetic material does not passively enter cells; thus, a delivery system is necessary. The emerging field of nano-gene delivery exploits the use of new materials and the properties that arise at the nanometre-scale to produce delivery vectors that can effectively deliver genetic material into a variety of different types of cells. The novel physicochemical properties of the new delivery vectors can be used to address the current challenges existing in nucleic acid delivery in vitro and in vivo. While there is a growing interest in nanostructure-based gene delivery, the field is still in its infancy, and there is yet much to discover about nanostructures and their physicochemical properties in a biological context.
\end{abstract}

We carried out an organised and focused search of bibliographic databases. Our results suggest that despite new breakthroughs in nanostructure synthesis and advanced characterization techniques, we still face many barriers in producing highly efficient and non-toxic delivery systems. In this review, we overview the types of systems currently used for clinical and biomedical research applications along with their advantages and disadvantages, as well as discussing barriers that arise from nano-scale interactions with biological material.

In conclusion, we hope that by bringing the far reaching multidisciplinary nature of nano-gene delivery to light, new targeted nanotechnology-bases strategies are developed to overcome the major challenges covered in this review.

Keywords: Gene delivery, transfection, gene therapy, nanotechnology, nano-gene delivery, biological therapy, bio-

medicine, nanomedicine.

\section{INTRODUCTION}

During the delivery of DNA or RNA, foreign genetic material is transferred intracellularly into target cells where it elicits a desired function. Delivery systems involve the use of either membrane-disruption or membrane-penetration in order to achieve delivery. Membrane-disruption involves induction of membrane permeabilization and the generation of gaps of sufficient size to allow passive passage through the mem-

*Address correspondence to this author at the Department of Zoology, Oxford University, OX26GG Oxford, UK;

E-mail: sonia.trigueros@zoo.ox.ac.uk

brane to the cytosol. Membrane-disruption systems are primarily physical, (Physical Systems) which induce transient discontinuities in the plasma membrane via mechanical, electrical, thermal, or optical means. These approaches can be thought of as permeabilization or uncoupled delivery systems. Alternatively, membranepenetration or coupled delivery systems (Biological and Chemical Systems) employ solid carriers to concurrently penetrate the membrane and introduce genetic material. Carrier-based approaches are comprised of viral and chemical systems, both of which have dimensions comparable to the molecular nano-scale of the components of target cells. Compared to viral vectors, nonviral gene delivery vectors are characterized by having low intrinsic immunogenicity, which is important for preventing a severe immune response in a clinical setting. In addition, nonviral vectors provide higher loading capacity and ease of fabrication [1,2].

Nanotechnology-based gene delivery is the division of nanomedicine concerned with the synthesis, characterization, 
and functionalization of nanomaterials for their use in targeted-gene delivery applications [1]. The field of nanotechnology has been active since before the year 2000, however it is only in the last few years that publications have begun appearing demonstrating the practical application of this technology showing results for both drug and gene delivery systems (Fig. 1). Significant progress in Nanotechnology and its application for drug and/or gene delivery has been made as a result of the converging efforts from different scientific disciplines (Fig. 2). Nanoparticles, the building blocks of nanotechnology, have been broadly defined as having at least one dimension at $100 \mathrm{~nm}$ or less. For biomedical applications, this definition has been expanded to include particles greater than $100 \mathrm{~nm}$, such as liposomes, in order to encompass particle sizes that take advantage of anatomical considerations, such as vascular gaps surrounding tumours [3-5].

Nanoparticles are solid structures prepared from natural or synthetic materials which are considered as good candidates as scaffolds for gene delivery systems for the following reasons: (1) they exist with a size comparable to cellular components such as proteins, lipids, and nucleic acids; (2) they have large surface to volume ratios which allow for the ability to have large numbers of surface functional groups; (3) they possess the ability for controllable absorption and release of small molecules and organic molecules; (4) nano-scale allows scientists to not only work at ever smaller dimensions but also utilize the unique physical, chemical, mechanical, and optical properties of materials that naturally occur at that the nano-scale. Nanoparticles, such as the quantum dots, gold nanoparticles and carbon nanotubes are particles generated to exploit the size-related properties inherent in the nano-scale (e.g.

conductivity, spectral properties, bio-distribution) $[4,6]$.

Until now, intracellular-delivery methods are typically not high-throughput, do not consistently disrupt cells, cause significant cellular injury and death, and are not very effective when delivering large or charged particles, such as DNA [7]. There are also a series of significant challenges which require overcoming, especial in a clinical setting, when considering the development of new delivery systems for genetic material.

These include synthesis, comparability and stability with extracellular compartments, transport and targeting, intracellular trafficking and final delivery to the point of action.
This review article gives an overview of current gene delivery systems, including physical, chemical, and biological. We describe recent advances with nanoscale delivery systems based on lipids, polyplexes, and other inorganic nanoparticles, as well as discussing emerging properties of nano-scale materials. Finally, we discuss the recognised challenges and current attempts to overcome and how novel combinations of approaches and materials may help to solve them.

\section{NANO-GENE DELIVERY SYSTEMS}

Nano-gene delivery systems take advantage of a host of different methods and delivery vectors to deliver genetic material into target cells. To be able to better understand the hurdles associated with the current generation of delivery systems as well as understand how to better improve them, it is necessary to first give a concise overview of the current delivery systems.

Gene delivery systems can broadly be split into those which are viral based, and those which are not (Table 1). Viral systems as the name suggests are ones which co-opt naturally occurring viruses such as lentivirus and adeno-associated virus for delivery of genetic material. The non-viral systems can be subdivided into physical systems (such as electroporation) and chemical systems, these, can be further separated into organic nano systems (e.g. polyplexes) and inorganic nano systems (e.g. gold nanoparticles).

Among the presented delivery methods, there are several ways to classify them. Viral and chemical systems, particularly organic ones, can be classified as nanoparticles due to their nano-scale size. However, despite being nanoscale the majority of the established systems do not benefit from the emergent nanoproperties which are present within the inorganic nano systems. For the purpose of this review organic and inorganic chemical systems but not viral systems will be considered as nano-gene delivery systems Table $\mathbf{1}$.

\subsection{Physical Delivery Systems}

The first delivery systems we will discuss are the non-nano physical systems. These systems take advantage of various physical properties for driving the entry of genetic material into cells. With genetic material in close proximity to cells, the plasma membrane is dis- 


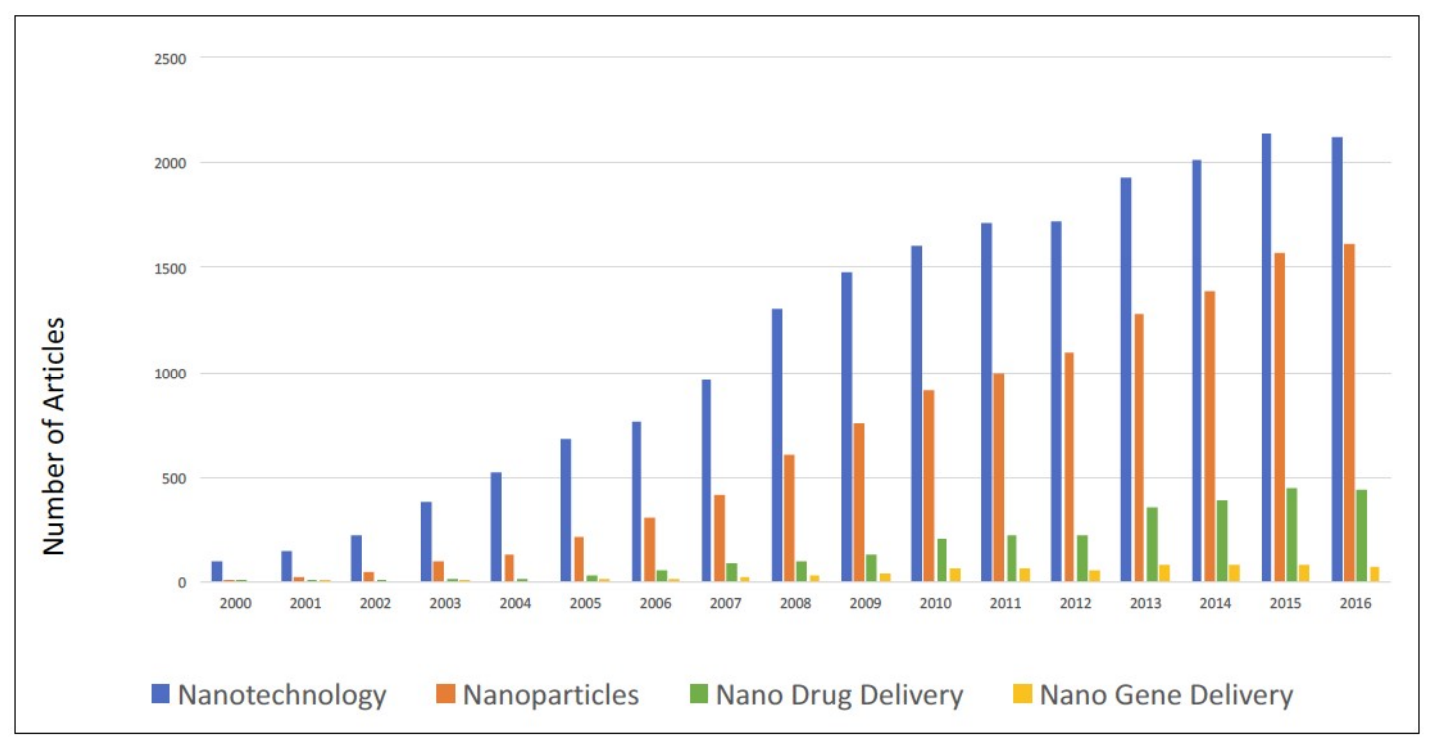

Fig. (1). Nanotechnology, Nanoparticles, drug delivery and gene delivery number of Publications per Year in the SCI 2000-2016. Data generated from Web of Science, an online search. Using a "title-abstract" search in SCI database for nanotechnology by keywords A-Analysis: document types: (article) 19,861 records. TOPIC: (Nanotechnology). Refined by: document types: (article) and topic: (nanoparticles) timespan: 2000-2016, 17441 records Refined by: document types: (article) and topic: (drug delivery) timespan: 2000-2016. 2734 records C-Refined by: document types: (article) and topic: (gene delivery) timespan: 2000-2016. 639 records indexes: sci-expanded, ssci, a\&hci, cpci-s, cpci-ssh, bkcis, bkci-ssh, esci, ccr-expanded, ic.
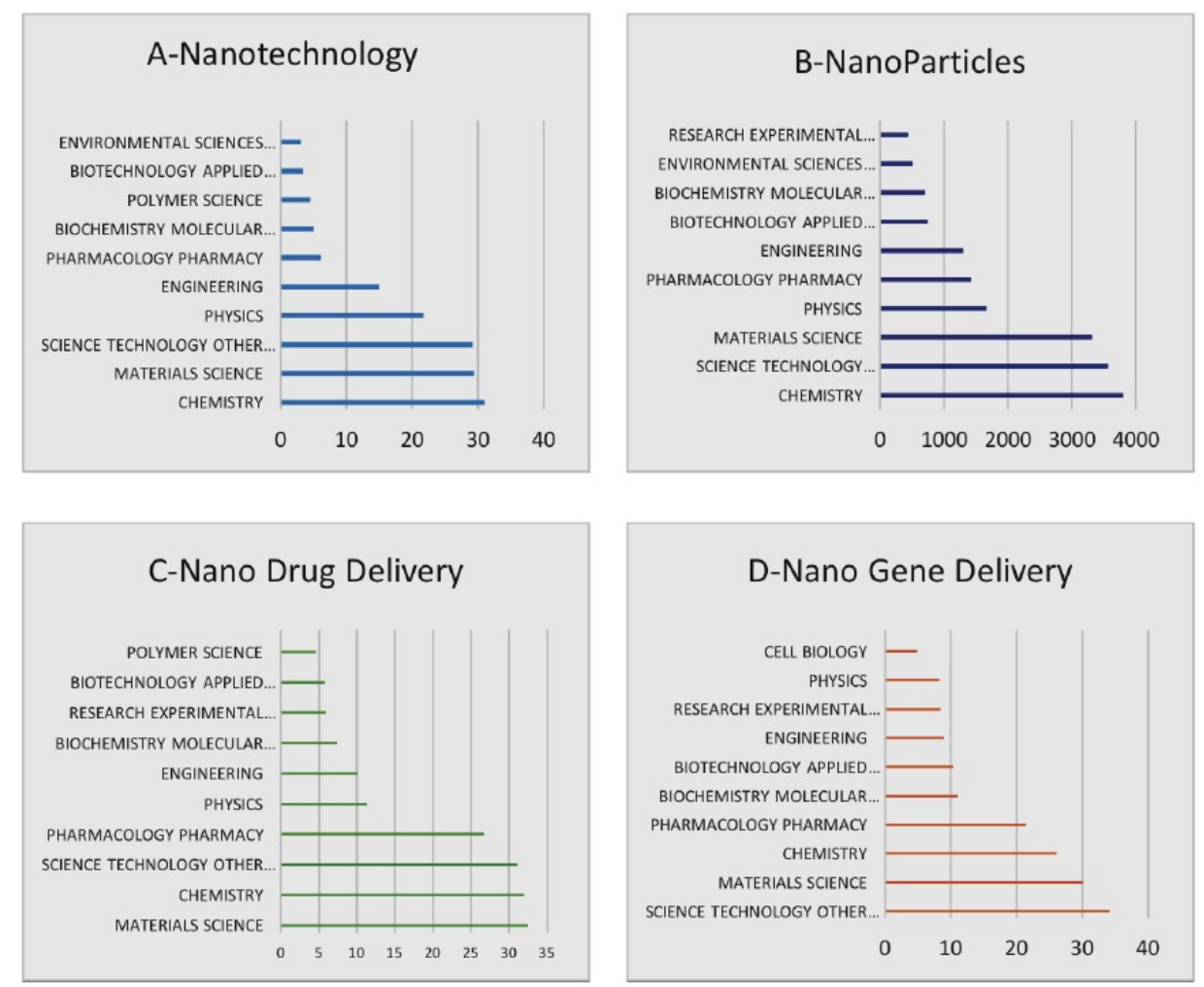

Fig. (2). Major Nanotechnology Research areas based on number of publications in the SCI 2000-2016. Data was generated from an online search in the Web of Science using a "title-abstract" search in SCI database for nanotechnology by keywords A-Analysis: document types: (article) and topic: (nanotechnology)19,861 records. B-Refined by: document types: (article) and topic: (nanoparticles) timespan: 2000-2016, 17441 records. C-Refined by: document types: (article) and topic: (drug delivery) timespan: 2000-2016, 2734records. D- Refined by: document types: (article) and topic: (gene delivery) timespan: 2000-2016, 639records. indexes: sci-expanded, ssci, a\&hci, cpci-s, cpci-ssh, bkci-s, bkcissh, esci, ccr-expanded, ic.

Table 1. Classification of current delivery systems covered in this review: Physical Systems, Chemical Systems and Biological Systems. Nano-gene delivery systems are included on Chemical Systems blue box. 


\begin{tabular}{|c|c|c|}
\hline \multicolumn{2}{|c|}{ Non-Viral Gene Delivery Systems } & Viral Gene Delivery Systems \\
\hline Physical Systems & Chemical Systems & Biological Systems \\
\hline $\begin{array}{ll}\text { - } & \text { Electroporation } \\
\text { - } & \text { Sonoporation } \\
\text { - } & \text { Magnetofection } \\
\text { - } & \text { Hydrodynamic gene transfer } \\
\text { - } & \text { Particle bombardment } \\
\text { - } & \text { Needle injection } \\
\text { - } & \text { Heat-shock transformation. }\end{array}$ & $\begin{array}{l}\text { Organic Nano Systems } \\
\text { - } \quad \text { Polyplexes } \\
\text { - } \quad \text { Lipolexes and LPD particles } \\
\text { Inorganic Nano Systems } \\
\text { - } \quad \text { Gold nanoparticles (AuNPs) } \\
\text { - } \quad \text { Magnetic Nanoparticles } \\
\text { - } \quad \text { Carbon Nanotubes (CNTs) } \\
\text { - } \quad \text { Quantum Dots (QDs) } \\
\text { - } \quad \text { Silica nanoparticles } \\
\text { - }\end{array}$ & 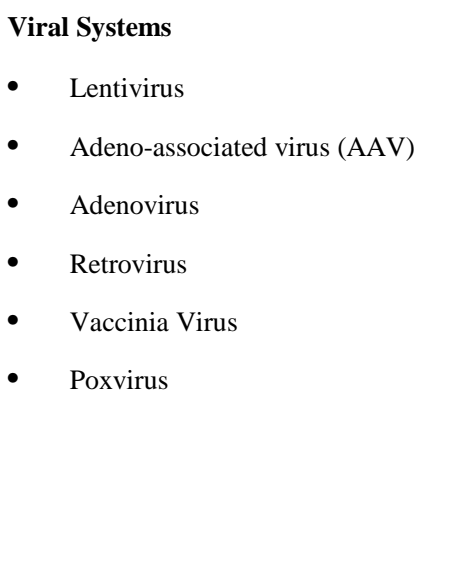 \\
\hline
\end{tabular}

rupted while concurrently allowing for the migration of the genetic material into the cell's cytosol. The most commonly used physical methods include the use of electric fields, sound waves, magnetic fields, high speed large volume delivery, direct high velocity force, direct injections, and heat shock.

The first two of these methods, which use controlled induction of electric fields and of ultrasound waves, are used for electroporation and sonoporation. Both of these are similar in that they produce transient membrane disruption and permeabilization to allow genetic material entry into the cell [8-10]. The next method makes use of magnetic fields and is the basis of magnetofection. This method involves combining genetic material and magnetic particles, which, through the presence of a magnetic field draws the complexes towards the target cells [11,12]. There is also the use of more directed systems. High velocity particles for direct force delivery of genetic material to target cells is one such system [13]. This involves the coating of small metallic particles with genetic material which are fired at high velocity towards the target cells, these particles then break their way into the cells where the genetic material is released. The second direct method is needle injection, of which there are two scales, larger volume directed injection of a genetic material containing solution into tissues and small scale microinjections such as used with mouse or xenopus eggs $[14,15]$. While this approach can be efficient for intramuscular delivery, it is less so for other tissues and for tumours. Moreover, this approach lacks the ability to target specific cell types when used in tissues. The last method, heat shock, is frequently used when introducing genetic material into bacterial cells which have artificially been made competent [16]. Competency is generally achieved though exposure of bacterial cells to $\mathrm{CaCl}_{2}$. To introduce genetic material cells are mixed with, for example, a plasmid which adheres to the bacterial membrane. The heat shock step triggers uptake of the genetic material into the bacterial cell.

These types of delivery systems avoid any possible problems with the immune-compatibility and present good tolerance from the body due to inherent low levels of toxicity. For these reasons, it will be beneficial to develop them further, and explore for new physical phenomena that could be exploited for gene delivery applications. In contrast to physical systems, chemical systems actively carry genetic material into their target cells. In order to reach their site of action, chemical methods use a carrier or vector which forms a complex with the genetic material. This complex provides protection for the cargo against nucleases, and helps actively gain access to the cytosol. There is a great research effort in the development of novel vectors as they can target tissues and specific cell types that are unreachable for physical methods.

\subsection{Chemical Delivery Systems}

For the purpose of this review we have broadly separated chemical systems, based on their components, into organic and inorganic nano systems. These systems have gathered incredible interest as they work at the nano-scale, and are safer and simpler options than viral vectors with distinct advantages such no limitations in transgene size. However, their delivery efficiency is low.

\subsection{Organic Nano Systems}

Polyplexes are nanoparticles that are created through chemical interactions between genetic material and various complexing molecules[17,18]. These molecules are often composed of specially designed polymers and cell-penetrating peptides, which make use of electrostatic interactions between positively charged moieties with negatively charged genetic material to induce the formation of nanoparticles[18,19]. The 
process of complex formation involves pushing the genetic material into a state that is as highly condensed as possible. The stoichiometry between the carrier's nitrogen content, the source for the majority of the positive charges, and the genetic material's phosphate ratio (N:P) plays an important role during the formation of polyplexes[20]. It is also important to take into consideration the composition of the medium used, as other negatively charged molecules can complete with those on the genetic material and adversely affect the ability of the polyplexs to form and maintain their integrity. Over time and under physiological conditions, polyplexs can disassemble releasing their contents, which is necessary for it to be functional within the cell. However, polyplex disassembly can also occur before it reaches the cell's cytosol, resulting in release and subsequent degradation of its contents in the medium[21]. Cationic particles have been used which reduced spontaneous disassembly, however, they can form aggregates after exposure to salts and biomolecules lowering their efficiency, extensive research is currently being undertaken to prevent this aggregation[22-24].

Lipoplexes and LPD nanoparticles are delivery vectors produced by taking advantage of the selfassembling properties of amphipathic lipids and liposomes into large supramolecular assemblies[17]. When the assembly is allowed to proceed in the presence of negatively charged nucleic acids, they become protected by being enveloped and located between the different cationic lipid layers within multi-layer lipid polyplexs. Depending on the original structure of the monomers, various final structures can be built, for instance DOPE forms an inverted Hexagonal $\mathrm{H}_{\text {II }}$ phase structure[17]. Lipid nanoparticles are produced in mixtures where the ratio of lipid per weight of genetic material is important for optimal delivery vector production. LPD differ from lipoplexes in terms of the physical state of the genetic material loaded within them, as it is condensed with polycations prior to encapsulation.

There are similar challenges when using these organic systems. Polyplexes, lipoplexes, and LPDs are all prone to aggregation in biological fluids over time, significantly reducing the time they are fully active. The grafting of hydrophilic polymers, such as polyethylene glycol (PEG) or poly ethyl ethylene phosphate (PEEP), to the surface of the nanoparticles is one avenue which has been explored to increase the duration their stability[23,25]. The nanoparticle's surface can also be functionalized with targeting moieties for improved pharmacokinetic properties. These organic nano systems have already proven their flexibility and clinical suitability, with lipoplexes and LPDs being used for the successful in vivo delivery of siRNA, RNA vaccines, and plasmid DNA which makes them attractive candidate for their continued used and development as delivery vectors [26-28]. Lipoplexes present low immunogenicity which is an attractive feature of their use, however, toxicity has been observed at high doses and they poses low transformation efficiency which subsequently requires larger amounts being used for efficient delivery[29].

\subsection{Inorganic Nano Systems}

Inorganic materials have recently emerged as a robust and versatile scaffold for nano-scale structures for use in gene delivery systems. These nanostructures are unique within the delivery systems we mention as they are generated from materials that at the nano-scale $(3100 \mathrm{~nm})$ have new physicochemical properties.

It is in part due to these properties that inorganic nanoparticles are exciting candidates for the basis of gene delivery systems. These materials have also been demonstrated to have low inherent toxicity, are available in a wide range of sizes and shapes, and offer the possibility for real-time tracking by various spectroscopic techniques. Inorganic nanoparticles also offer an appealing set of properties for practical applications including scalability in synthesis, facile functionalization, chemical and thermal stability[30].

There are three different strategies being employed for using inorganic nanoparticles as gene delivery vectors. The first is the use of positively charged inorganic nanoparticles to form complexes with negatively charged genetic material. The second strategy involves the direct conjugation of genetic material onto the inorganic nanoparticles via the use of a linker. And the third strategy involves the use of cationic amphiphilic polymers grown from the surface of the nanoparticles to help the formation complexes between inorganic nanoparticles and genetic material. Gold nanoparticles, magnetic nanoparticles, carbon nanotubes, quantum dots and silica nanoparticles are the most commonly used in these strategies[31].

The use of nanoparticle systems confers several advantages such as short time of transfection, easy preparation, wide availability, rich functionality, high transfection efficiency, potential capability for targeted delivery and controlled release of the genetic material cargo. Although most of them are instable, toxic and nonbiocompatible [6,32].

Gold nanoparticles (AuNPs) are highly versatile like all metallic nanoparticles. It has been shown that metallic nanoparticles are able to link with different type of molecules, including different types of polymers and genetic material such as oligos, siRNA, and plasmid DNA. Therefore they are good potential delivery systems with useful properties that rise from this particular metal[33].

Gold nanoparticles are currently the most widely used variety of inorganic nanoparticle. This is due to their versatility, in being able to be produced within a defined range of nanometre sizes and shapes, their photonic properties, highly tuneable surface chemistry, high biocompatibility, ease of synthesis, well-defined surface chemistry, and facile 
molecular imaging by FRET. Gold nanoparticles can be functionalized easily with positive charges molecules such as amino acids, cationic peptides, and amine-containing molecules. These positively charged molecules act as a linker to improve DNA and RNA binding. Other methods to improve binding have explored the gold-thiol chemical bonds for conjugation genetic material. However, the major drawback of these methods is that the synthesis process requires multiple steps to generate surface functionalisation. To overcome this problem a controlled synthesis of polyethyleneimine (PEI) coated AuNPs (AuPEI-NPs) was achieved for siRNA

delivery[34]. It has been found that in the case of AuPEI-NPs as a transfection vector, the smaller the size of the nanoparticle the lower the agglomeration state inside the cell and the faster endosomal escape is. While spherical nanoparticles generally exhibit poor performance as delivery vectors, nanorods have been found to be a more efficient vector. Understanding the beneficial properties of different nanoparticle sizes and morphology is a challenge that needs to be explored in order to design and generate the most effective delivery system. Currently, several studies are investigating different AuNPs functionalization strategies in order to improve the delivery efficiency of genetic material[31].

Magnetic Nanoparticles (MNPs) such as those made with a magnetic iron (II, III) oxide (Fe3O4) core which is coated with a biocompatible layer and superparamagnetic iron oxide nanoparticles are commercially available. MNPs have been studied as potential delivery vectors for genetic material due to their small size which is capable of penetrating into cells and also because these nanoparticles can be used with high precision by the use of an external magnetic field. Superparamagnetic iron oxide nanoparticles (SPION) are small synthetic $\gamma$-Fe2O3 or $\mathrm{Fe} 3 \mathrm{O} 4$ particles with a core size of $<10 \mathrm{~nm}$ with an organic or inorganic coating. The term "superparamagnetism" is used to infer an analogy between the behaviour of the small magnetic moment of a single paramagnetic atom and that of the much larger magnetic moment of a nanosized magnetic particle which arises from the coupling of many atomic spins. When the magnetic field is removed, the nanoparticles do not show magnetic interaction; this quality makes them especially useful. Particles such as liposomes containing magnetic oxide particles in their cavities are called magnetosomes, whereas aggregates consisting of organic compounds (dextran, starch, etc.) in combination with nano-scale iron oxide particles are called superparamagnetic beads or agglomerates[35]. It has been described that to avoid potential toxic effects on cells, MNPs, can be coated with a shell of a stable and biocompatible material such as silica (SiO2)[3639]

Protocols developing the use of novel magnetic vectors for gene delivery use different strategies. One of the most successful strategies in terms of enhanced efficiency of gene transfer uses the self-assembling properties of nanoparticles with viral and non-viral vectors[40]. Also, it has been shown that an oscillating magnetic field generated by magnets positioned below an in vitro cell culture plate promotes more efficient endocytosis via mechanical stimulation[41]. It has also been reported the nanoparticles fabrication of magnetic biomimetic contrast agents which have a dual function: effective for therapeutic gene delivery and magnetic detection. These nanoparticles are made of a functionalised iron oxide core and the shell can be made of an inert gold layer or silica. On top of that, the outmost corona is typically made up of targeting ligands attached to the inert metallic nanoshell[42].

Carbon Nanotubes (CNTs) have been widely explored for potential biomedical applications since the first publication of CNTs in 1991[43]. These nanostructures are rolled-up sheets of carbon atoms. Carbon nanotubes can be either single-walled (SWCNTs) or multi-walled (MWCNTs), each of which is known to have a different electron arrangement and as a result, different properties. However, the shared unique properties of both types of CNT allow for their potential use in various biomedical devices and therapies. Some of the most common properties of these materials include the ability to absorb near-infra-red light and generate heat, the ability to deliver drugs in a cellular environment, their light weight, and chemical stability. However, toxicity of pure CNTs represents a major challenge for clinical application. Despite the drawbacks of in vitro studies, they have been invaluable in identifying important toxicity factors, such as size, shape, purity, and functionalisation, the latter of which can attenuate CNT toxicity. Functionalization of CNTs surface can be done by one of two different protocols: by oxidation of the CNT in acid conditions and 1,3-dipolar cycloaddition reaction obtaining covalently linked functionality, or, by hydrophobic stacking between the CNTs and another non-polar ring to produce noncovalent conjugates. The result is the generation of water soluble CNTs that can be used for biomedical applications. Both SWCNTs and MWCNTs have also been found to form stable complexes with plasmid DNA allowing their potential use as gene delivery vectors[31]. Their use in in vitro gene transfer has been investigated as CNTs are considered as excellent nanomaterials for gene and drug delivery as the offer an uptake pathway independent of endocytosis of mammalian cells[44].

Quantum Dots (QDs) are semiconducting particles of only a few nanometres in size (radius of 1-20nm). Optical and electronic properties at their lower nanoscale size differ from those of larger particles. Many types of quantum dots will emit light of specific frequencies when electricity or light is applied to them. These frequencies can be precisely tuned by changing the dots' size, shape or material. For instance, larger QDs (radius of 5-6 nm) emit longer wavelengths resulting in emission colours such as orange or red. Smaller QDs (radius of 2-3 nm) emit shorter wavelengths resulting in colours like blue and green, although the specific colours and sizes vary depending on the exact composition of the QD[45]. 
The use of QDs as siRNA carries has proven to be one of the most efficient method to achieve siRNA delivery. Because QDs are luminous they can be used as fluorescent probes for living cells, and in vivo cancer targeting and diagnostic[31]. Although the cytotoxicity of QDs limits their biological application, several potential organic-hybrid modifications been reported to overcome this problem[46]. Also, Li et al. demonstrated that water-soluble and cysteamine protected (CdTe) QDs can form large complexes with plasmid DNA by electrostatic attractions which can be used as potential in vitro gene delivery vectors[46]. Studies on quantum dot toxicity are focused on cadmium containing particles which has yet to be demonstrated in animal models after physiologically relevant dosing[47]. In cell cultures studies, QD toxicity suggests that their toxicity may be derived from multiple factors including their physicochemical characteristics and environment. Assessing their potential toxicity is complex as these factors include properties such as QD size, charge, concentration, chemical composition, capping ligands, and also on their oxidative, mechanical and photolytic stability[48].

Silica nanoparticles (SNP) can be found as pure silica, organically modified silica, hollow silica nanoparticles, nanotubes, and mesoporus silica nanoparticles (MSN). They are amenable to diverse polymerisation of, and functionalization with various polymers. Their flexible hydrophobic and hydrophilic properties make MSN a promising material for biomedical applications, such as delivering drugs or biological molecules (siRNA or DNA) to target cells or tissues. MSN have great accessibility in permeable structures for encapsulation of medications and genes. MSN have an extensive surface region to stack biomacromolecules and they are stable and biocompatible. It has been shown that MSN with small a pore size can be utilized for plasmid delivery and MSN with positive charges on their surface are suitable for siRNA delivery. Different functionalization of silica NPs of different morphology, size, and shape are currently being tested for potential cytotoxicity and gene transfection capabilities. Nanorods of 300nm length proved to be the most efficient as gene carrier[31].

\subsection{Biological Delivery Systems: Viral Gene Delivery}

Biological systems are those which involved hijacking of naturally occurring viruses for use as delivery vectors. Viral vectors are more efficient than non-viral vectors for DNA delivery but can present a significant risk to patients when used in a clinical setting, while non-viral carriers are inherently safer than viral carriers[6].

Viruses are naturally occurring pathogenic nanoparticles that lack the ability to auto-replicate. In order to survive, viruses have to find and infect a host, after which it hijacks their molecular machinery to replicate. Viruses have developed very sophisticated mechanisms to inject their genome into target cells. Due to their ability to deliver their genome into different cells and tissues, viruses can be seen as biological gene delivery vectors.

The vast diversity of viruses is reflected in the number of viruses with different tissue and cell tropism; infection mechanisms, toxicity and immunogenicity; genomic characteristics, and genetic material fate within the host. For example, there is growing interest in researching lentiviruses, adenoviruses, adenoassociated viruses, retroviruses, poxvirus, and vaccinia virus. This diversity can be exploited to produce vital nanoparticles that can specifically deliver, and transfer a gene of interest, after they are engineered to overcome their toxicity.

There are currently many clinical trials using viral vectors for gene delivery, mostly adenoviruses and retroviruses; however, toxicity, and immunogenic issues are challenging their success[49]. In this review, we will focus on the two most commonly used viral vectors in research and with potential for gene delivery clinical applications.

Lentivirus are retroviruses with the ability to infect dividing and non-dividing cells[49]. They exhibit desirable traits for gene delivery such as their ability to integrate their genome within the host's chromosomes, preventing the transgene dilution after cell division. Lentiviruses are enveloped RNA viruses that encapsulate two copies of positive RNA within its capsid. The viral genome is retrotranscribed into DNA by the viral reverse transcriptase and integrated in the host genome by an integrase.

Transforming a lentivirus into a transgene delivery vector requires the separation of its viral cycle into two stages: the production of the essential proteins for the viral cycle, and the transfer of a defective viral vector to the nucleus. Transgenecontaining viral particles have the necessary proteins for wild type virus reconstitution removed, and replaced by the gene of interest. As the lentiviral vector lacks the necessary proteins for assembling the lentiviral particles, they have to be provided in trans. This can be accomplished via packaging cell lines which stably express the required viral proteins or transient transfection with multiple DNA plasmids.

Lentiviral gene delivery vectors can be engineered to target different tissues. The envelope proteins determine the tropism of the virus. However, this can be changed by the expression of targeting ligands as well as pseudotyping, which consists of expressing other viral glycoproteins for altering the natural tropism[5052]. For instance, lentiviruses pseudotyped with the vesicular stomatitis virus's glycoprotein (VSVG) are more stable, and can infect a large range of cell types, as seen in robust hematopoietic gene transfer[53,54].

There are safety concerns with the use of lentiviral particles for gene delivery. It is known that they prefer to insert into regions of the genome which are actively transcribed, favouring regions downstream from regulatory sequences[55,56]. To prevent potential genotoxicity from the long terminal repeats' (LTR) enhancer regions self- 
inactivating (SIN) vectors have been developed to prevent gene activation downstream the integration site[53]. Other approaches to deal with potential genotoxicity are the integration-deficient lentiviral vectors, whose integration abilities are abolished retaining their genome in episomes[57].

Adeno-associated virus (AAV) are small, naked, single stranded DNA viruses without an associated human disease, despite infecting a large portion of the population[58]. These $25 \mathrm{~nm}$ diameter viruses have a genome approximately $5 \mathrm{~kb}$ in size which can be exchanged for a transgene, provided the LTRs are preserved. As their name implies, they require the presence of a helper virus, normally the adeno viruses, but it has been shown that herpesvirus or baculovirus can also play this role. Similarly, to lentiviral vectors, there have been efforts to avoid using virus coinfections, favouring multiple plasmid transfections as production methods. There are many AAV serotypes, AAV2 being the most studied. Consequently, AAV based vectors can be readily pseudotyped with other serotype's proteins and engineered to increase their tissue selectivity[50,52,59,60]

While viruses are very efficient for gene transfer, their repeated clinical use is limited due to their recognition by the immune system, transgene size restrictions or methylationdriven silencing. Complement system can recognise and inactivate viruses. For instance lentiviruses are particularly sensitive to complement recognition, resulting in loss of infectivity[61]. There are less immune-sensitive alternatives such as adenoassociated viruses; however, they have a small capsid and viral titres drop sharply as the size of the transgene increases[58]. Transgene size is extremely limiting due to the large size necessary for human genes. Moreover, there are advantages to delivering large inserts. For instance, long-term transgene expression is close to endogenous levels when the whole genomic locus is used[62]. While the ability to integrate their genome is valuable, integrations events can result in lower expression levels when compared to their endogenous versions, due to methylation-driven silencing[56]. Viral delivery vectors are promising systems, but they are hindered by their immunogenicity, limited transgene encapsulation, and toxicity.

\section{CHALLENGES FOR CURRENT NANO-GENE DELIVERY SYSTEMS}

There are strengths and weakness to all delivery systems, some of the specifics have already been mentioned. However, there are also a host of obstacles which all delivery systems face and have to be overcome in order to successfully deliver genes. Taking these into account when considerations are made for designing new or improved delivery systems will be key for the development of future delivery systems. Here we describe several of gene delivery barriers from synthesis to eliciting a function. These challenges also apply to viruses; however, we will focus in nano-gene delivery systems.

\subsection{Synthesis}

Nano-gene delivery systems are complex to produce and require multiple steps. Depending on the type of vector, they face different challenges during production, especially in obtaining material at a sufficient scale for use. Scaling-up all aspects during production of a completed nano-gene delivery system is technically complex and is expensive[63]. New synthesis methodologies and strategies need to be devised in order to make these systems more accessible by bringing the cost down. For example, producing clinical-grade biological vectors, such as viruses, is expensive. Similarly, chemical methods, particularly inorganic vectors, are only stable at low concentrations; thus, large scale/volume nano-gene delivery system have to be produced for research or potential clinical applications[64,65]. However, manufacturing these vectors is not the only synthesis challenge, loading them with high efficiency with a gene of interest is not trivial.

Efficiently introducing nucleic acids into cells using the delivery systems discussed above represents a major technical challenge. There is a correlation between size and loading efficiency, smaller genes more easily loaded and delivered than larger genes. However, as mentioned before, human genes are large, and this inherently lowers the efficiency with which they can be loaded. For example, AAVs have a finite genome size they can fit within their capsid, and increasing the transgene size beyond its optimal limit is associated with a sharp decrease in viral particle production[66]. While chemical methods can be loaded with nucleic acids with no theoretical size limit, their already low delivery efficiency is reduced further with increases in transgene size[67].

Taking all this into consideration, developing new manufacturing methodologies, as well as loading strategies, will be crucial for the successful application of nano-gene delivery systems in both the bench and the clinic.[63-65,67] (Fig. 3.I).

\subsection{Biological Considerations}

Gene delivery systems have to reach their cellular target in order to deliver their content, and in doing so they will have to interact with many biological macromolecules, as well as cellular entities. These interactions arise from the content and biological properties of the environment, and the mechanism that govern particle recognition, uptake, and once in the cytoplasm intracellular trafficking [68]. Understanding these phenomena provides insights into the necessary considerations required for improving future design and formulations of nano-gene delivery systems. We will cover the most relevant biological barriers with the greatest impact on gene delivery.

\subsubsection{Extracellular Components}

Nanoparticles have a large surface to volume ratio with which they can interact with molecules in a solution; these 
interactions can affect the stability of delivery systems. Solutes which come into contact with the nanoparticle's surface experience a vast range of forces associated with the physicochemical properties of the particle's surface [22]. Among many things, this can result in the unwanted accumulation of biomolecules on the nanoparticle. Proteins in solution can screen the nanoparticle's surface and get adsorbed onto the nanoparticle. This phenomenon is known as protein corona formation, and it is highly dynamic, by which the composition of the protein layers on the nanoparticle changes over time. The physicochemical properties of the nanostructure and bio-molecules are modulated by the biological dispersant (an aqueous solution of biological origin in which the nanoparticles are dispersed in solution), which will have a great impact on the composition of the corona. Solvation forces, Van der Waals interactions, the nanoparticle's and protein's surface charges are involved in the corona formation 
and dynamics. The nanoparticle decoration with proteins and biomolecules provides it with a "biological identity" which plays a major role in delivery performance, circulation and clearance by the immune system, and toxicity[69]. Moreover, these interactions can also trigger the aggregation of the nanoparticles, resulting in a physical state vastly different from the originally produced monodisperse nanoparticle with completely different physicochemical properties and delivery fate[24].

This problem has been routinely tackled by shielding nanoparticles with hydrophilic polymers[23,25]. This approach increases their stability; however, at the cost of reducing the interactions between the nanoparticle and their target. Stimulus-responsive surface modifications have been created for the purpose of shedding the surface polymer when favourable conditions for interaction are met [70,71]. Exploring the dynamics of the corona and the effects on nanoparticle fate will be crucial for improving nanoparticle design (Fig. 3.II).

\subsubsection{Transport}

There are barriers to the use of systemicadministered nanoparticles that hinders their stability while circulating to finally reach their target tissue. The vascular endothelium is comprised of endothelial cells, which tightly adhere to one another, forming a difficult to penetrate barrier. For a delivery system to be useful in a clinical setting nanoparticles must be able to pass, intact, through this endothelial barrier[72]. This is challenging as endothelial cells are connected via adherens and tight junctions, leaving available only gaps of $\sim 3 \mathrm{~nm}$ for cellular transport[73,74]. Transcytosis through caveolae vesicles is an alternative passing route. These trafficking vesicles are approximately $70 \mathrm{~nm}$ in size; however, they can also fuse to form channel-like structures known as VesiculoVacuolo Organelles (VVO), directly connecting both sides of the endothelial cell[75-77]. Not all fenestrations are the same size, the Blood-Brain Barrier (BBB) junctions are tighter, as small molecules like sucrose are not allowed through; and the liver, part of the reticuloendothelial system (RES) contains larger ones, resulting in the organ with the highest accumulation of nanoparticles[78-80]. Tumours are also known to have leaky vasculature with large fenestrations, which promotes the accumulation of nanoparticles[81,82]. This phenomenon is known as the enhanced permeability and retention (EPR) effect. These physical constraints and mechanisms would have to be exploited in order to pass through the vascular endothelium and our target tissues.

Along with the problems associated with vascular endothelium, phagocytic cells from the RES are responsible for nanoparticle clearance from circulation. Kupffer cells from the liver sinusoids and splenic macrophages have developed highly sophisticated phagocytic abilities that allow them to internalize nanoparticles. These cells will need to be avoided in order to increase survival time during circulation before degradation[80]. Once the vascular endothelium is passed, nanoparticles need to diffuse through the Extracellular Matrix (ECM) in order to reach their target[83].

There is an interest in elucidating all parameters that play a key role in nanoparticle circulation, and tissue accumulation (Fig. 3.III).

\subsubsection{Targeting}

Cellular surface markers are used to identify particular cells types of interest for targeting purposes. Depending on the nanoparticle's mode of entry, it would be necessary to target receptors that are readily internalized upon targeting ligandreceptor recognition. Many nanoparticles require endocytosis in order to reach the cell's interior. Ideally, these receptors would be exclusive to the target of interest, they would be expressed in high enough numbers to ensure the interaction between the nanoparticle and the receptor, they will be internalized and recycled at a high rate, and they will have known high affinity ligands that can be exploited as targeting moieties. Unfortunately, no such receptors exist, and, generally, there is a trade-off between exclusivity and copy number [84].

Modifying the surface of nanoparticles with antibodies against transmembrane proteins on target cells has proven effective for enhanced uptake. For instance, lipoplexes and LPDs grafted with PEG coronas can increase their interactions with cells of interest by functionalizing their surfaces with antibodies [67]. This strategy can help them overcome the low interaction abilities produced by their PEG coronas. There are many other targeting molecules available used for targeting a molecular payload, such as aptamers, sugars, small molecules, peptides, scFv or DARPins [85-90].

As discussed above, it is challenging to identify surface markers that are exclusive; thus, functionalizing nanoparticles with targeting ligands will not result in total accumulation in the targeted site. The resultant accumulation will be determined by the nanoparticle's ability to overcome all the barriers discussed in this section. Therefore, considering other aspects of nanoparticle design could yield higher nanoparticle accumulation in the intended site of action (Fig. 3.IV).

\subsubsection{Internalization and Trafficking}

Nanoparticles are required to undergo cellular internalization, so that their cargo can be released to exert their effect on cytoplasmic and nuclear targets. The majority of nanoparticles require internalization after they have successfully interacted with cells via their surface receptors. This triggers the nanoparticle internalization through endocytosis [91,92].

Endocytosis comprises a series of complex molecular processes that coordinate the formation of invaginations of the cellular membrane, which results in the acquisition of fluid from the extracellular medium along with any nanoparticles attached to the membrane. There are several mechanisms 
through which endocytosis occurs, internalization via clathrin-coated pits is the most studied. Caveolae dependent, CLIC/GEEC mechanism, and alternative endocytosis pathways are other processes available to the cell for internalization. Some bio-macromolecules are linked specifically to a particular internalization pathway; however, different entry routes can be used to accommodate the necessity of the cell at any given moment. Alongside endocytosis, macropynocytosis is another mechanism used to uptake large amounts of fluid, and particles[92,93].

Once the nanoparticles have been internalized, they reach a complex network of membranous compartments used to sort the incoming material. These compartments are known as endosomes, and they are differ in size, and composition[94,95]. The journey starts at the cellular periphery in the early endosomes (EEs), and can then take paths towards one of two final destinations, the lysosomes, where the nanoparticles will be degraded, or the extracellular medium after they are recycled and shuttled back out, as they ride the vesicles used to return the receptors to the cell surface. As the nanoparticles travel further into the cell, they are carried to the perinuclear region into the multivesicular bodies (MVB). These compartments are filled with intralumenal vesicles (ILVs) formed from invaginations of the MVBs, and are released to the extracellular medium as exosomes, shuttling nucleic acids, and proteins with them into neighbouring cells[96]. Then, the nanoparticles are taken to the lysosomes for degradation. This is accomplished by the heavy presence of $\mathrm{pH}$-activated hydrolases.

The luminal $\mathrm{pH}$ on the different endocytic compartments becomes more acidic as the nanoparticles reach the lysosomes. EEs start at $\mathrm{pH}$ 6-6.5, whereas the lysosomes are at $\mathrm{pH}$ 4.5-5, a condition needed to activate the luminal hydrolases. Considering the highly degradative environment of the endosome/lysosomes after internalisation, recent work has been focused on strategies to promote endosomal escape or lysosomal avoidance. Membrane-destabilizing peptides, incorporation of cationic polymers in nanoparticles and/or enable their internalisation by caveolae-mediated endocytosis. The caveolae-mediated mechanism of endocytosis results in the formation of caveosomes of neutral $\mathrm{pH}$, which in certain instances have been shown to bypass lysosomes[97]. Similarly, nanoparticles coated with cellular membranes have shown the ability to avoid lysosomes altogether[98]. It would be vital to understand how these mechanisms affect gene delivery so the relevant trafficking pathways for nanoparticle internalization and successful delivery of the gene cargo can be identified (Fig. 3.V).

\subsubsection{Endosomal Escape}

Genetic material needs to reach the nucleus in order to carry out its function, but before this can happen, delivery vectors need to escape the endosomal compartments to access the cytosol and move towards the nucleus[99]. It was noted that synthetic carriers would have poor transfections efficiencies due to their inability to escape the endosomes[100]. Polyplexes, lipoplexes and LPDs, and metallic nanoparticles have been engineered to disrupt the endosomal membrane and gain access to the cytosol; however, there are many strategies that can be used to accomplish it[23,100,101].

Due to the vast chemical space available to create cationic polymers, it is widely known that polypexes can be engineered to escape the endosomes. The most common escaping strategy used is the "proton sponge" effect[23]. Cationic polymers with titratable groups buffer the entry of protons in the endosomal compartment as a result of maturation acidification. Consequently, the polymer behaves as a proton sponge. The normal proton influx is compensated by a complementary ion influx; however, the polymer is capturing protons from solution. As a result, the influx of compensating ions keeps increasing, until the solute concentration in the endosomal lumen produces an osmotic shock strong enough to transiently disrupt the compartment's membrane. The proton sponge phenomenon is somewhat controversial as there are reports in the literature that Polyethylenimine (PEI) can escape without altering the endosome's $\mathrm{pH}$, which suggests alternative escaping mechanisms[102]. On the other hand, there are reports that state otherwise, and conclude that proton buffering is necessary for cationic dendrimers to escape the endosomes[103].

An alternative escaping route for polymeric nanoparticles is through polymer solubilisation-drive osmotic shock[104,105]. Polymerics vesicles (polymersomes) made out of poly(2-(methacryloyloxy) ethylphosphorylcholine)-copoly(2-(diisopropylamino) ethylmethacrylate) (PMPCPDPA) disassemble in the endosomes due to the luminal acidification, which solubilises about half million ionic species. This translates into an osmotic shock that perforates the endosomal membrane.

Other polyplexing molecules, such as cellpenetrating peptides, can even be classified according to their mechanisms to access the cells[19]. This variety of mechanisms brings to light the value of polyplexes, and allows choosing from a pool of possible carriers that could meet the requirements for gene delivery.

Lipoplexes and LPDs generate membrane disruption through the mixing of their and the endosome's lipids. The phase transition of some lipids enhances the disruption of endosomes. Lipids such as dioleoylphosphatidylethanolamine (DOPE) are known to arrange into an inverted hexagonal phase. This phenomenon is correlated with enhanced transfection efficiency; thus, many lipid-based carriers are designed to trigger the formation of such phase upon interaction with the anionic lipids present in the endosomal membrane[106]. While the geometrical structure of the lipids can influence the delivery efficiency, the charge density can as well[17]. Lamellar lipoplexes increase their transfection 
efficiency as a function of charge density, most probably due to favourable DNA-lipid interactions, as well as the promotion of membrane fusion between the liposome and the endosomal membrane.

Some inorganic nanoparticles, such as carbon nanotubes, can access the cytosol in an endosomeindependent manner[107]. Nevertheless, many types of inorganic nanoparticles have to be equipped with molecules that enhance endosomal escape in order to overcome this barrier [101] (Fig. 3.VI).

\subsubsection{DNA Release}

Once the endosomal barrier is overcome, it is crucial to release the genetic material from the carrier in order to be active $[108,109]$. The release of the nucleic acid cargo is generally mediated by the interaction of the nanoparticle with other anionic species that compete for the carrier and positively charged ions that destabilise the condensed state of DNA [110]. Chemically modified polymers for inducing DNA release upon gaining access to the cytosol have been developed as candidates for controlled gene-release [21]. Nevertheless, keeping the DNA condensed in the cytosol results in higher movement rates towards the nucleus, and increased protection [111]. Many gene delivery platforms are cell-cycle dependent; in fact, it has been shown that many DNA molecules need to wait until the nuclear membrane is dissolved during mitosis to gain access to the nucleus [111]. The size of the condensed DNA molecule is generally too big to go through the nucleopore; however, it is still possible for it to access the nucleus, only with significant less efficiency [111,112] (Fig. 3.VII).

\section{CONCLUSION}

In summary, we have selectively reviewed the latest and most promising advances in nano-gene- delivery systems. To make significant progress in engineering the next generation of nano-gene delivery systems, we highlighted the advantages and challenges of the most recently developed systems. We also bring to light that although having lower efficiency in DNA transfection, chemical non-viral vectors are relatively easy to prepare, less immunogenic and oncogenic, are less prone to recombination events, and have no limitation on the size of a transferred gene. The capability to incorporate plasmid DNA into nanoparticles with low toxicity demonstrates a new era in pharmacotherapy for delivering genetic material selectively to tissues and cells. However, their use in clinical applications is still debatable. To date, the Food and Drug Administration (FDA) has not approved any nano-gene delivery system because of the unknown long-term toxicity and the low gene transfection efficiency of nanomaterials in vivo. To overcome these challenges, new nano-gene delivery systems will need to be sitting at the functional intersection of physical, inorganic/organic chemical, and viral systems taking the best aspects of all of them: efficiency, biocompatibility, nano-scale size, and adaptable physicochemical properties. While also, incorporating new designs for working towards specific cell and/or tissue targeting and overcoming several of the aforementioned challenges.

There is a great need for further development of genedelivery vectors. In gene therapy, for instance, there are enough scientific evidences indicating that the lack of a full gene delivery systems is one of the major steps hindering progress towards successful treatment of a diseases including correcting genetic disorders. 

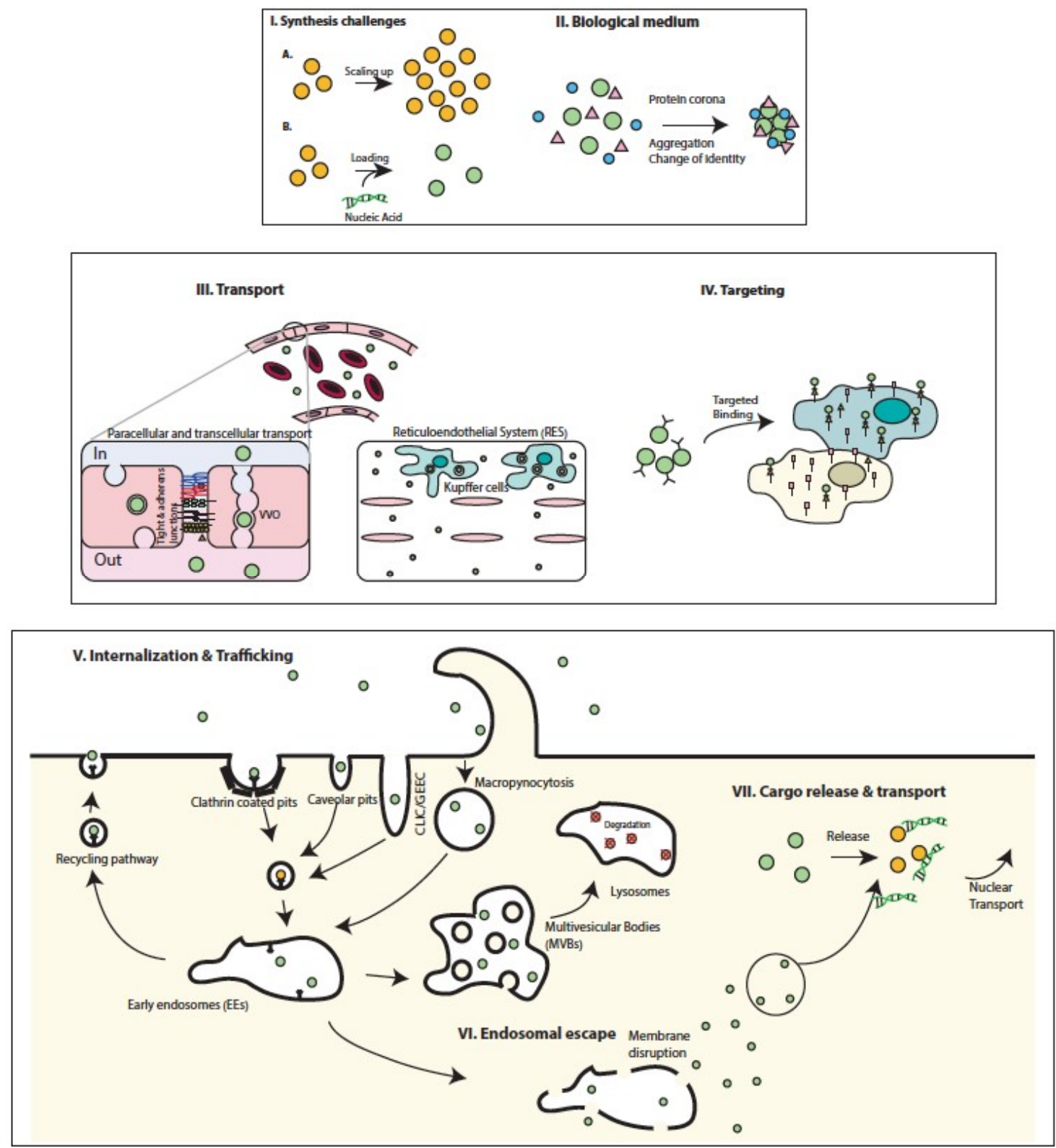

Fig. (3). Current Challenges on Nano-gene delivery vectors: Top box. Challenges associated with the production of nanogene delivery systems and their stability in biological media. I. Synthesis challenges. It highlights the problems with scaling-up vector production, and nucleic acid loading. II. Challenges in the biological medium. Nano-gene delivery systems can aggregate and become useless due to bio-macromolecule surface adsorption. Middle box. Current biological challenges that has to be overcome in order to reach specific cells. III Nano-gene delivery system transport to their target site. Transcellular transport from circulation to tissues is limited to small particles; thus, cellular transport mechanisms have to be exploited. Furthermore, nano-gene delivery systems have to evade the RES in order to avoid clearance. IV. Targeting challenges. There are no unique molecular targets at the cell's surface that can be used to specifically reach one cell type. Other cell-types may express those same receptors, resulting impartial, non-targeted accumulation of the nano-gene delivery system. Bottom box: Challenges associated with intracellular cargo trafficking towards the nucleus. V. Internalization pathways. There are several pathways for nano-gene delivery system for entering the cell. After internalization in the endosomal compartments, nano-gene delivery systems have to avoid recycling to the extracellular medium, as well as degradation in the lysosomes. VI. Endosomal escape into the cytosol. Nano-gene delivery systems have to bring their cargo into the cells cytosol, and they have to escape from the endosomal compartments to accomplish it. There are several strategies for disrupting temporarily the endosome's membrane, providing an escaping route for the nano-gene delivery system. VII. Cytosolic release of the nucleic-acid cargo and transport to the cell's nucleus. Nano-gene delivery systems have to free their cargo in the right cellular compartment in order to allow the nucleic acid molecule to reach their final destination.

The most suitable vector must be able to deliver genes to the appropriated tissue or cells in the body in a specific, safe 
and effective manner. In addition, nanogene delivery is also currently being developed for in vivo real time characterization of genetic activity of selected genes with the hope to provide a better understanding of the biological mechanisms involved in different diseases.

We have summarised in this review the first steps and the most relevant up-to date designs at the nanoscale. However, nanotechnology and its application to gene delivery is still in its infancy. Emerging nanomaterials with versatile properties such as optical, piezoelectric, thermal, electrochemical, enzyme mimicking etc. are still being developed. With the proper combination of novel properties, nanotechnology will bring to us new and exciting tools to explore the design of innovative, efficient, accurate, stable in different biological fluids and/or different temperatures and most importantly, low toxicity nano-gene delivery vectors.

Beyond the outline of recent advances summarized in this paper, it is easy to imagine that a deeper development of nanotechnology, nanomaterials and nanogene-delivery will, undoubtedly, make a significant impact on biomedical research and translational medicine.

\section{CONSENT FOR PUBLICATION}

Not applicable.

\section{CONFLICT OF INTEREST}

The authors declare no conflict of interest, financial or otherwise.

\section{ACKNOWLEDGEMENTS}

All individuals listed as authors have contributed substantially to the collections and discussions of the reported published work. A.G.G has received financial support through the "la Caixa” Fellowship Grant for PostGraduate Studies, "la Caixa” Banking Foundation, Barcelona, Spain. TLD was supported by EPA Cephalosporin Fund CF289 and ST by EPSRC IAA D4D00620. S.T. also acknowledges support from the Oxford Martin School.

We are grateful to Professor Gemma Marfany and Professor Peter Holland for critical reading of the manuscript.

\section{REFERENCES}

[1] Keles, E.; Song, Y.; Du, D.; Dong, W.-J.; Lin, Y. Recent Progress in Nanomaterials for Gene Delivery Applications. Biomater. Sci. 2016, 4, 1291-1309.

[2] Stewart, M. P.; Sharei, A.; Ding, X.; Sahay, G.; Langer, R.; Jensen, K. F. In Vitro and Ex Vivo Strategies for Intracellular Delivery. Nature 2016, 538, 183-192.

[3] Garnett, M. C.; Kallinteri, P. Nanomedicines and Nanotoxicology: Some Physiological Principles. Occup. Med. (Chic. Ill). 2006, 56, 307-311.

[4] Stern, S. T.; McNeil, S. E. Nanotechnology Safety Concerns Revisited. Toxicol. Sci. 2008, 101, 4-21.

[5] Mansoori, G. A.; Fauzi Soelaiman, T. A. Nanotechnology - An Introduction for the Standards Community. J. ASTM Int. 2005, 2, 121.
[6] Dizaj, S. M.; Jafari, S.; Khosroushahi, A. Y. A Sight on the Current Nanoparticle-Based Gene Delivery Vectors. Nanoscale Res. Lett. 2014, 9, 252.

[7] Toner, M. Gene Delivery: Suddenly Squeezed and Shocked. Nat. Biomed. Eng. 2017, 1, 47.

[8] André, F.; Mir, L. M. DNA Electrotransfer: Its Principles and an Updated Review of Its Therapeutic Applications. Gene Ther. 2004, 11 Suppl 1, S33-S42.

[9] Kleinberger, T. Anticancer Genes. Adv Exp Med Biol 2014, 818, 6197.

[10] Newman, C. M. H.; Bettinger, T. Gene Therapy Progress and Prospects: Ultrasound for Gene Transfer. Gene Ther. 2007, 14, 465475.

[11] Dobson, J. Gene Therapy Progress and Prospects: Magnetic Nanoparticle-Based Gene Delivery. Gene Ther. 2006, 13, 283-287.

[12] Suda, T.; Liu, D. Hydrodynamic Gene Delivery: Its Principles and Applications. Mol. Ther. 2007, 15, 20632069.

[13] Al-Dosari, M. S.; Gao, X. Nonviral Gene Delivery: Principle, Limitations, and Recent Progress. AAPS J. 2009, 11, 671.

[14] Wolff, J. A.; Budker, V. The Mechanism of Naked DNA Uptake and Expression. Adv. Genet. 2005, 54, 1-20.

[15] Herweijer, H.; Wolff, J. A. Progress and Prospects: Naked DNA Gene Transfer and Therapy. Gene Ther. 2003, 10, 453-458.

[16] Van Die, I. M.; Bergmans, H. E. N.; Hoekstra, W. P. M. Transformation In Escherichia Coli: Studies On The Role Of The Heat Shock In Induction Of Competence. Microbiology 1983, 129, 663-670.

[17] de Ilarduya, C. T.; Sun, Y.; Düzgüneş, N. Gene Delivery by Lipoplexes and Polyplexes. Eur. J. Pharm. Sci. 2010, 40, 159-170.

[18] Pack, D. W.; Hoffman, A. S.; Pun, S.; Stayton, P. S. Design and Development of Polymers for Gene Delivery. Nat Rev Drug Discov 2005, 4, 581-593.

[19] Kauffman, W. B.; Fuselier, T.; He, J.; Wimley, W. C. Mechanism Matters: A Taxonomy of Cell Penetrating Peptides. Trends Biochem. Sci. 2015, 40, 749-764.

[20] Gary, D. J.; Min, J.; Kim, Y.; Park, K.; Won, Y. Y. The Effect of N/P Ratio on the in Vitro and in Vivo Interaction Properties of Pegylated poly[2-(Dimethylamino)ethyl Methacrylate]-Based siRNA Complexes. Macromol. Biosci. 2013, 13, 1059-1071.

[21] Oupický, D.; Parker, A. L.; Seymour, L. W. Laterally Stabilized Complexes of DNA with Linear Reducible Polycations: Strategy for Triggered Intracellular Activation of DNA Delivery Vectors. J. Am. Chem. Soc. 2002, 124, 8

9

[22] Nel, A. E.; Mädler, L.; Velegol, D.; Xia, T.; V Hoek, E. M. Somasundaran, P.; Klaessig, F.; Castranova, V.; Thompson, M. Understanding Biophysicochemical Interactions at the Nano-bio Interface. Nat. Mater. 2009, 8, 543-557.

[23] Huang, L.; Guo, S. Nanoparticles Escaping RES and Endosome: Challenges for siRNA Delivery for Cancer Therapy. J. Nanomater. 2011, 2011.

[24] Rausch, K.; Reuter, A.; Fischer, K.; Schmidt, M. Evaluation of Nanoparticle Aggregation in Human Blood Serum. Biomacromolecules 2010, 11, 2836-2839.

[25] Schöttler, S.; Becker, G.; Winzen, S.; Steinbach, T.; Mohr, K.; Landfester, K.; Mailänder, V.; Wurm, F. R. Protein Adsorption Is Required for Stealth Effect of Poly(ethylene Glycol)-and Poly(phosphoester)- Coated Nanocarriers. Nat Nano 2016, 11, 372377.

[26] Semple, S. C.; Akinc, A.; Chen, J.; Sandhu, A. P.; Mui, B. L.; Cho, C. K.; Sah, D. W. Y.; Stebbing, D.; Crosley, E. J.; Yaworski, E.; et al. Rational Design of Cationic Lipids for siRNA Delivery. Nat. Biotechnol. 2010, 28, 172-176.

[27] Kranz, L. M.; Diken, M.; Haas, H.; Kreiter, S.; Loquai, C.; Reuter, K. C.; Meng, M.; Fritz, D.; Vascotto, F.; Hefesha, H.; et al. Systemic RNA Delivery to Dendritic Cells Exploits Antiviral Defence for Cancer Immunotherapy. Nature 2016, 534, 396-401.

[28] Heyes, J.; Palmer, L.; Chan, K.; Giesbrecht, C.; Jeffs, L.; Maclachlan, I. Lipid Encapsulation Enables the Effective Systemic Delivery of Polyplex Plasmid DNA. Mol. Ther. 2007, 15, 713-720.

[29] Liu, C.; Zhang, N. Chapter 13 - Nanoparticles in Gene Therapy: Principles, Prospects, and Challenges. In Nanoparticles in 
Translational Science and Medicine; Science, A. V. B. T.-P. in M. B. and T., Ed.; Academic Press, 2011; Vol. Volume 104, pp. 509562.

[30] Loh, X. J.; Lee, T.-C.; Dou, Q.; Deen, G. R. Utilising Inorganic Nanocarriers for Gene Delivery. Biomater. Sci. 2016, 4, 70-86.

[31] Loh, X. J.; Lee, T.-C.; Dou, Q.; Roshan Deen, G. Utilising Inorganic Nanocarriers for Gene Delivery. Biomater. Sci. 2016, 4, 70-86.

[32] Ramos-Perez, V.; Cifuentes, A.; Coronas, N.; de Pablo, A.; Borrós, S. Modification of Carbon Nanotubes for Gene Delivery Vectors. In Nanomaterial Interfaces in Biology: Methods and Protocols; Bergese, P.; Hamad-Schifferli, K., Eds.; Humana Press: Totowa, NJ, 2013; pp. 261-268.

[33] Stevenson, A. P.; Blanco Bea, D.; Civit, S.; Antoranz Contera, S.; Iglesias Cerveto, A.; Trigueros, S. Three Strategies to Stabilise Nearly Monodispersed Silver Nanoparticles in Aqueous Solution. Nanoscale Res. Lett. 2012, 7, 151.

[34] Lee, Y.; Lee, S. H.; Kim, J. S.; Maruyama, A.; Chen, X.; Park, T. G. Controlled Synthesis of PEI-Coated Gold Nanoparticles Using Reductive Catechol Chemistry for siRNA Delivery. J. Control. Release 2011, 155, 3-10.

[35] Neuberger, T.; Schöpf, B.; Hofmann, H.; Hofmann, M.; Von Rechenberg, B. Superparamagnetic Nanoparticles for Biomedical Applications: Possibilities and Limitations of a New Drug Delivery System. J. Magn. Magn. Mater. 2005, 293, 483-496.

[36] Correa-Duarte, M. a.; Giersig, M.; Kotov, N. a.; LizMarzán, L. M. Control of Packing Order of Self-Assembled Monolayers of Magnetite Nanoparticles with and without $\mathrm{SiO} 2$ Coating by Microwave Irradiation. Langmuir 1998, 14, 6430-6435.

[37] Lu, Y.; Yin, Y.; Mayers, B. T.; Xia, Y. Modifying the Surface Properties of Superparamagnetic Iron Oxide Nanoparticles through a Sol-Gel Approach. Nano Lett. 2002, 2, 183-186.

[38] Yoon, T.-J.; Kim, J. S.; Kim, B. G.; Yu, K. N.; Cho, M.-H.; Lee, J.K. Multifunctional Nanoparticles Possessing A "Magnetic Motor Effect” for Drug or Gene Delivery. Angew. Chemie Int. Ed. 2005, 44, 1068-1071.

[39] Majidi, S.; Sehrig, F. Z.; Samiei, M.; Milani, M.; Abbasi, E.; Dadashzadeh, K.; Akbarzadeh, A. Magnetic Nanoparticles: Applications in Gene Delivery and Gene Therapy. Artif. Cells, Nanomedicine, Biotechnol. 2016, 44, 1186-1193.

[40] Mykhaylyk, O.; Sanchez-Antequera, Y.; Tresilwised, N.; Döblinger, M.; Thalhammer, S.; Holm, P. S.; Plank, C. Engineering Magnetic Nanoparticles and Formulations for Gene Delivery. J. Control. Release 2010, 148, e63-e64.

[41] Fouriki, A.; Dobson, J. Oscillating Magnet Array-Based Nanomagnetic Gene Transfection of Human Mesenchymal Stem Cells. Nanomedicine 2013, 9, 989-997.

[42] Bikram, M. Ultrasmall Superparamagnetic Iron Oxide Nanoparticles and Uses Thereof, 2010.

[43] Iijima, S. Helical Microtubules of Graphitic Carbon. Nature 1991, 354, 56-58.

[44] Pantarotto, D.; Singh, R.; McCarthy, D.; Erhardt, M.; Briand, J. P.; Prato, M.; Kostarelos, K.; Bianco, A. Functionalized Carbon Nanotubes for Plasmid DNA Gene Delivery. Angew. Chemie - Int. Ed. 2004, 43, 5242-5246.

[45] Jain, K. K. The Handbook of Nanomedicine; 1st ed.; Humana Press, 2008.

[46] Li, D.; Li, G.; Guo, W.; Li, P.; Wang, E.; Wang, J. GlutathioneMediated Release of Functional Plasmid DNA from Positively Charged Quantum Dots. Biomaterials 2008, 29, 2776-2782.

[47] Tsoi, K. M.; Dai, Q.; Alman, B. A.; Chan, W. C. W. Are Quantum Dots Toxic? Exploring the Discrepancy between Cell Culture and Animal Studies. Acc. Chem. Res. 2013, 46, 662-671.

[48] Hardman, R. A Toxicologic Review of Quantum Dots: Toxicity Depends on Physicochemical and Environmental Factors. Environ. Health Perspect. 2006, 114, 165-172.

[49] Kotterman, M. A.; Chalberg, T. W.; Schaffer, D. V. Viral Vectors for Gene Therapy: Translational and Clinical Outlook. Annu. Rev. Biomed. Eng 2015, 17, 63-89.

[50] Waehler, R.; Russell, S. J.; Curiel, D. T. Engineering Targeted Viral Vectors for Gene Therapy. Nat. Rev. Genet. 2007, 8, 573-587.

[51] Cronin, J.; Zhang, X.-Y.; Reiser, J. Altering the Tropism of Lentiviral Vectors through Pseudotyping. Curr. Gene Ther. 2005, 5, 387-398.
[52] Schaffer, D. V; Koerber, J. T.; Lim, K.-I. Molecular Engineering of Viral Gene Delivery Vehicles. Annu. Rev. Biomed. Eng. 2008, 10, 169-194.

[53] Montini, E.; Cesana, D.; Schmidt, M.; Sanvito, F.;

Bartholomae, C. C.; Ranzani, M.; Benedicenti, F.; Sergi, L. S.; Ambrosi, A.; Ponzoni, M.; et al. The Genotoxic Potential of Retroviral Vectors Is Strongly Modulated by Vector Design and Integration Site Selection in a Mouse Model of HSC Gene Therapy. J. Clin. Invest. 2009, 119, 964-975.

[54] Wu, C.; Dunbar, C. E. Stem Cell Gene Therapy: The Risks of Insertional Mutagenesis and Approaches to Minimize Genotoxicity. Front. Med. 2011, 5, 356-371.

[55] Biffi, A.; Bartholomae, C. C.; Cesana, D.; Cartier, N.; Aubourg, P.; Ranzani, M.; Cesani, M.; Benedicenti, F.; Plati, T.; Rubagotti, E.; et al. Lentiviral Vector Common Integration Sites in Preclinical Models and a Clinical Trial Reflect a Benign Integration Bias and Not Oncogenic Selection. Blood 2011, 117, 5332 LP-5339.

[56] Herbst, F.; Ball, C. R.; Tuorto, F.; Nowrouzi, A.; Wang, W.; Zavidij, O.; Dieter, S. M.; Fessler, S.; van der Hoeven, F.; Kloz, U.; et al. Extensive Methylation of Promoter Sequences Silences Lentiviral Transgene Expression during Stem Cell Differentiation in Vivo. Mol. Ther. 2012, 20, 1014-1021.

[57] Wanisch, K.; Yáñez-muñoz, R. J. Integration-Deficient Lentiviral Vectors : A Slow Coming of Age. Mol. Ther. 2009, 17, 1316-1332.

[58] Samulski, R. J.; Muzyczka, N. AAV-Mediated Gene Therapy for Research and Therapeutic Purposes. Annu. Rev. Virol. 2014, 1, $427-$ 451.

[59] Ai, J.; Li, J.; Gessler, D. J.; Su, Q.; Wei, Q.; Li, H.; Gao, G. AdenoAssociated Virus Serotype rh.10 Displays Strong Muscle Tropism Following Intraperitoneal Delivery. Sci. Rep. 2017, 7.

[60] Foust, K. D.; Nurre, E.; Montgomery, C. L.; Hernandez, A.; Chan, C. M.; Kaspar, B. K. Intravascular AAV9 Preferentially Targets Neonatal Neurons and Adult Astrocytes. Nat Biotech 2009, 27, 5965.

[61] DePolo, N. J.; Reed, J. D.; Sheridan, P. L.; Townsend, K.; Sauter, S. L.; Jolly, D. J. VSV-G Pseudotyped Lentiviral Vector Particles Produced in Human Cells Are Inactivated by Human Serum. Mol. Ther. 2000, 2, 218-222.

[62] Lufino, M. M.; Edser, P. A.; Wade-Martins, R. Advances in HighCapacity Extrachromosomal Vector Technology: Episomal Maintenance, Vector Delivery, and Transgene Expression. Mol. Ther. 2008, 16, 1525.

[63] Paliwal, R.; Babu, R. J.; Palakurthi, S. Nanomedicine Scaleup Technologies: Feasibilities and Challenges. AAPS PharmSciTech 2014, 15, 1527-1534.

[64] Tsuzuki, T. Commercial Scale Production of Inorganic Nanoparticles. Int. J. Nanotechnol. 2009, 66, 567-578.

[65] Vauthier, C.; Bouchemal, K. Processing and Scale-up of Polymeric Nanoparticles. Pharm. Res. 2009, 26, 10251058.

[66] Clément, N.; Grieger, J. C. Manufacturing of Recombinant AdenoAssociated Viral Vectors for Clinical Trials. Mol. Ther. Methods Clin. Dev. 2016, 3, 16002.

[67] Xu, L.; Anchordoquy, T. Drug Delivery Trends in Clinical Trials and Translational Medicine: Challenges and Opportunities in the Delivery of Nucleic Acid-Based Therapeutics. J. Pharm. Sci. 2011, 100, 38-52.

[68] Blanco, E.; Shen, H.; Ferrari, M. Principles of Nanoparticle Design for Overcoming Biological Barriers to Drug Delivery. Nat. Biotechnol. 2015, 33, 941-951.

[69] Salvati, A.; Åberg, C.; Dawson, K. A.; Monopoli, M. P.; Åberg, C.; Salvati, A.; Dawson, K. A.; Aberg, C.; Salvati, A.; Dawson, K. A. Biomolecular Coronas Provide the Biological Identity of Nanosized Materials. Nat. Nanotechnol. 2012, 7, 779-786.

[70] Cui, C.; Yu, P.; Wu, M.; Zhang, Y.; Liu, L.; Wu, B.; Wang, C. X.; Zhuo, R. X.; Huang, S. W. Reduction-Sensitive Micelles with Sheddable $\{$ PEG $\}$ Shells Self-Assembled from a Y-Shaped Amphiphilic Polymer for Intracellular Doxorubicine Release. Colloids Surfaces B Biointerfaces 2015, 129, 137-145.

[71] Gao, W.; Langer, R.; Farokhzad, O. C. Poly(ethylene Glycol) with Observable Shedding. Angew. Chemie - Int. Ed. 2010, 49, 65676571.

[72] Yuan, S.; Rigor, R. Regulation of Endothelial Barrier Function. In Colloquium Series on Integrated Systems Physiology: From 
Molecule to Function; Morgan \& Claypool Life Sciences, San Rafael (CA), 2010.

[73] Wallez, Y.; Huber, P. Endothelial Adherens and Tight Junctions in Vascular Homeostasis, Inflammation and Angiogenesis. Biochim. Biophys. Acta - Biomembr. 2008, 1778, 794-809.

[74] Bazzoni, G.; Dejana, E. Endothelial Cell-to-Cell Junctions: Molecular Organization and Role in Vascular Homeostasis. Physiol. Rev. 2004, 84, 869-901.

[75] El-Fadaly, A. B.; Kummer, W. Endothelial VesiculoVacuolar Organelles, Pockets and Multi-Layered Fenestrated Lamellae in the Capillaries of the Mouse Carotid Body. Ann Anat 2005, 187, 333344.

[76] Dvorak, A. M.; Feng, D. The Vesiculo-Vacuolar Organelle (VVO): A New Endothelial Cell Permeability Organelle. J. Histochem. Cytochem. 2001, 49, 419-431.

[77] Feng, D.; Nagy, J. A.; Dvorak, H. F.; Dvorak, A. M. Ultrastructural Studies Define Soluble Macromolecular,

Particulate, and Cellular Transendothelial Cell Pathways in Venules, Lymphatic Vessels, and Tumor-Associated Microvessels in Man and Animals. Microsc. Res. Tech. 2002, 57, 289-326.

[78] Banks, W. A. From Blood-brain Barrier to Blood-brain Interface: New Opportunities for CNS Drug Delivery. Nat.

Rev. Drug Discov. 2016, 15, 275-292.

[79] Rubin, L. L.; Staddon, J. M. The Cell Biology of the BloodBrain Barrier. Annu. Rev. Neurosci 1999, 22, 11-28.

[80] Alexis, F.; Pridgen, E.; Molnar, L. K.; Farokhzad, O. C. Factors Affecting the Clearance and Biodistribution of Polymeric Nanoparticles. Mol. Pharm. 2008, 5, 505-515.

[81] Baluk, P.; Hashizume, H.; M, D. M. Cellular Abnormalities of Blood Vessels as Targets in Cancer. Curr. Opin. Genet. Dev. 2005, 15, 102111.

[82] Matsumoto, Y.; Nichols, J. W.; Toh, K.; Nomoto, T.; Cabral, H.; Miura, Y.; James Christie, R.; Yamada, N.; Ogura, T.; Kano, M. R.; et al. Vascular Bursts Enhance Permeability of Tumour Blood Vessels and Improve Nanoparticle Delivery. Nat. Nanotechnol. 2016, 11, 533538 .

[83] Jain, R. K. Transport of Molecules in the Tumor Interstitium : A Review Transport of Molecules in the Tumor Interstitium: A Review1. Cancer Res. 1987, 47, 3039-3051.

[84] Srinivasarao, M.; Galliford, C. V; Low, P. S. Principles in the Design of Ligand-Targeted Cancer Therapeutics and Imaging Agents. Nat. Rev. Drug Discov. 2015, 14, 203-219.

[85] Kanwar, J. R.; Roy, K.; Kanwar, R. K. Chimeric Aptamers in Cancer Cell-Targeted Drug Delivery. Crit. Rev. Biochem. Mol. Biol. 2011, 46, 459-477.

[86] Wu, D. Q.; Lu, B.; Chang, C.; Chen, C. S.; Wang, T.; Zhang, Y. Y.; Cheng, S. X.; Jiang, X. J.; Zhang, X. Z.; Zhuo, R. X. Galactosylated Fluorescent Labeled Micelles as a Liver Targeting Drug Carrier. Biomaterials 2009, 30, 1363-1371.

[87] Liu, Y.; Li, K.; Pan, J.; Liu, B.; Feng, S. S. Folic Acid Conjugated Nanoparticles of Mixed Lipid Monolayer Shell and Biodegradable Polymer Core for Targeted Delivery of Docetaxel. Biomaterials 2010, 31, 330-338.

[88] Oller-Salvia, B.; Sá Nchez-Navarro, M.; Giralt, E.; Teixidó, M. Blood-brain Barrier Shuttle Peptides: An Emerging Paradigm for Brain Delivery. Chem. Soc. Rev. 2016, 45, 4631-4852.

[89] Richards, D. A.; Maruani, A.; Chudasama, V. Antibody Fragments as Nanoparticle Targeting Ligands: A Step in the Right Direction. Chem. Sci. 2017, 8, 63-77.

[90] Boersma, Y. L.; Plückthun, A. DARPins and Other Repeat Protein Scaffolds: Advances in Engineering and Applications. Curr. Opin. Biotechnol. 2011, 22, 849-857.

[91] Doherty, G. J.; McMahon, H. T. Mechanisms of Endocytosis. Annu. Rev. Biochem. 2009, 78, 857-902.

[92] Mercer, J.; Schelhaas, M.; Helenius, A. Virus Entry by Endocytosis. Annu. Rev. Biochem. 2010, 79, 803-833.

[93] Hussain, M.; Fazil, U. T.; Ong, S. T.; Latha, M.; Chalasani,
S.; Low, J. H.; Kizhakeyil, A.; Mamidi, A.; Fang, C.; Lim, H.; et al. GapmeR Cellular Internalization by Macropinocytosis Induces Sequence-Specific Gene

Silencing in Human Primary T-Cells. Sci. Rep. 2016, 6.

[94] Huotari, J.; Helenius, A. Endosome Maturation. EMBO J. 2011, 30, 3481-3500.

[95] Scott, C. C.; Vacca, F.; Gruenberg, J. Endosome Maturation, Transport and Functions. Semin. Cell Dev. Biol. 2014, 31, 2-10.

[96] Colombo, M.; Raposo, G.; Théry, C. Biogenesis, Secretion, and Intercellular Interactions of Exosomes and Other Extracellular Vesicles. Annu. Rev. Cell Dev. Biol 2014, 30, 255-289.

[97] Chou, L. Y. T.; Ming, K.; Chan, W. C. W. Strategies for the Intracellular Delivery of Nanoparticles. Chem. Soc. Rev. 2011, 40, 233-245.

[98] Parodi, A.; Quattrocchi, N.; van de Ven, A. L.; Chiappini, C.; Evangelopoulos, M.; Martinez, J. O.; Brown, B. S.; Khaled, S. Z.; Yazdi, I. K.; Enzo, M. V.; et al. Synthetic Nanoparticles Functionalized with Biomimetic Leukocyte Membranes Possess Cell-like Functions. Nat. Nanotechnol. 2013, 8, 61-68.

[99] Varkouhi, A. K.; Scholte, M.; Storm, G.; Haisma, H. J. Endosomal Escape Pathways for Delivery of Biologicals. J. Control. Release 2011, 251, 220-228.

[100] Stewart, M. P.; Lorenz, A.; Dahlman, J.; Sahay, G. Challenges in Carrier-Mediated Intracellular Delivery: Moving beyond Endosomal Barriers. Wiley Interdiscip. Rev. Nanomedicine Nanobiotechnology 2016, 8, 465-478.

[101] Ma, D. Enhancing Endosomal Escape for Nanoparticle Mediated siRNA Delivery. Nanoscale 2014, 6, 6415-6425.

[102] Benjaminsen, R. V; Mattebjerg, M. A.; Henriksen, J. R.; Moein Moghimi, S.; Andresen, T. L. The Possible "Proton Sponge" Effect of Polyethylenimine (PEI) Does Not Include Change in Lysosomal pH. Mol. Ther. 2013, 21, 149-157.

[103] Freeman, E. C.; Weiland, L. M.; Meng, W. S. Modeling the Proton Sponge Hypothesis: Examining Proton Sponge Effectiveness for Enhancing Intracellular Gene Delivery through Multiscale Modeling. J. Biomater. Sci. Polym. Ed. 2013, 24, 398-416.

[104] Du, J.; Tang, Y.; Lewis, A. L.; Armes, S. P. pH-Sensitive Vesicles Based on a Biocompatible Zwitterionic Diblock Copolymer. J. Am. Chem. Soc. 2005, 127, 17982-17983.

[105] Lomas, H.; Massignani, M.; Abdullah, K. A.; Canton, I.; Presti, C. Lo; Macneil, S.; Du, J.; Blanazs, A.; Madsen, J.; Armes, S. P.; et al. Non-Cytotoxic Polymer Vesicles for Rapid and Efficient Intracellular Delivery. Faraday Discuss. 2008, 139, 143-159.

[106] Du, Z.; Munye, M. M.; Tagalakis, A. D.; Manunta, M. D. I.; Hart, S. L. The Role of the Helper Lipid on the DNA Transfection Efficiency of Lipopolyplex Formulations. Sci. Rep. 2014, 4.

[107] Kostarelos, K.; Lacerda, L.; Pastorin, G.; Wu, W.; Wieckowski, S.; Luangsivilay, J.; Godefroy, S.; Pantarotto, D.; Briand, J.-P.; Muller, S.; et al. Cellular Uptake of Functionalized Carbon Nanotubes Is Independent of Functional Group and Cell Type. Nat. Nanotechnol. 2007, 2, 108-113.

[108] Schaffer, D. V.; Fidelman, N. A.; Dan, N.; Lauffenburger, D. A. Vector Unpacking as a Potential Barrier for ReceptorMediated Polyplex Gene Delivery. Biotechnol. Bioeng. 2000, 67, 598-606.

[109] Medina-Kauwe, L.; Xie, J.; Hamm-Alvarez, S. Intracellular Trafficking of Nonviral Vectors. Gene Ther. 2005, 12, 1734-1751.

[110] Cohen, R. N.; van der Aa, M. A. E. M.; Macaraeg, N.; Lee, A. P.; Szoka, F. C. Quantification of Plasmid DNA Copies in the Nucleus after Lipoplex and Polyplex Transfection. J. Control. Release 2009, 135, 166-174.

[111] Elouahabi, A.; Ruysschaert, J. M. Formation and Intracellular Trafficking of Lipoplexes and Polyplexes. Mol. Ther. 2005, 11, 336347.

[112] Dean, D. A.; Dean, B. S.; Muller, S.; Smith, L. C. Sequence Requirements for Plasmid Nuclear Import. Exp. Cell Res. 1999, 253, 713-722. 
DISCLAIMER: The above article has been published in Epub (ahead of print) on the basis of the materials provided by the author. The Editorial Department reserves the right to make minor modifications for further improvement of the manuscript.

PMID: 29308734 